UNIVERSITY OF GOTHENBURG

SCHOOL OF BUSINESS, ECONOMICS AND LAW

WORKING PAPERS IN ECONOMICS

No 351

\title{
Risk Aversion and Expected Utility of Consumption over Time
}

\author{
Olof Johansson-Stenman
}

April 2009

ISSN 1403-2473 (print)

ISSN 1403-2465 (online) 


\title{
Risk Aversion and Expected Utility of Consumption over Time
}

\author{
Olof Johansson-Stenman \\ Department of Economics, University of Gothenburg, Box 640, SE-40530 Göteborg, Sweden \\ Phone: + 4631 7862538,Fax: + 4631 7861043, E-mail: Olof.Johansson@economics.gu.se
}

\begin{abstract}
The calibration theorem by Rabin (2000) implies that seemingly plausible smallstake choices under risk imply implausible large-stake risk aversion. This theorem is derived based on the expected utility of wealth model. However, Cox and Sadiraj (2006) show that such implications do not follow from the expected utility of income model. One may then wonder about the implications for more applied consumption analysis. The present paper therefore expresses utility as a function of consumption in a standard life cycle model, and illustrates the implications of this model with experimental small- and intermediate-stake risk data from Holt and Laury (2002). The results suggest implausible risk aversion parameters as well as unreasonable implications for long term risky choices. Thus, the conventional intertemporal consumption model under risk appears to be inconsistent with the data.
\end{abstract}

Key words: Expected utility of income, expected utility of final wealth, dynamic consumption theory, asset integration, time inconsistency, narrow bracketing

JEL classification: D81, D91

Acknowledgement: I am grateful for very constructive comments from two anonymous referees, an advisory editor, Martin Dufwenberg, Kjell Arne Brekke, Thomas Aronsson, Frank Heinemann, Robert Östling, Richard Thaler, Peter Wakker, Fredrik Carlsson, Matthias Sutter and seminar participants at Umeå University, as well as for financial support from the Swedish Research Council and the Swedish International Development Cooperation Agency (Sida). 


\section{Introduction}

How well expected utility (EU) theory describes human behavior in general, including in small- and intermediate-stake gambles, has recently been discussed intensively. At the core is what expected utility is expressed as a function of. This note provides a simple extension of some important aspects of this discussion to a life cycle setting where people derive utility from consumption (instead of wealth or payoffs), and illustrates this with numerical implications based on experimental data from Holt and Laury (2002).

Rabin (2000) presents an important theoretical contribution in terms of a calibration theorem that implies conclusions of the following kind: "If for all wealth levels an expected utility maximizing person turns down a 50-50 lose \$100/gain \$200 gamble, he would also turn down a 50-50 lose \$200/gain \$20,000 gamble.” While it may seem plausible that some people would turn down the first gamble (for all wealth levels), it seems much less reasonable to turn down the second. According to Rabin and Thaler (2001, 206): “Even a lousy lawyer could have you declared legally insane for turning down this bet.”1 An important feature of this calibration theorem is that it does not assume anything regarding the functional form of the utility function. However, the "for all wealth levels" part of the theorem is important. Although one can derive less extreme versions without this assumption, one must still assume

\footnotetext{
${ }^{1}$ Given that "expected utility" refers to "expected utility of wealth," it is actually straightforward to derive an even stronger conclusion, as follows: "If for all wealth levels an expected utility maximizing person turns down a 50-50 lose \$100/gain \$200 gamble, he would also turn down a 50-50 lose \$200/gain infinity gamble.” Let $K$ denote the (cardinal) gain in utility $U$ from a wealth increase from $w$ to $w+200$, where $w$ is initial wealth. Then if the individual turns down a 50-50 lose \$100/gain \$200 gamble, it follows by concavity that the utility loss from a wealth change from $w$ to $w-200$ is at least $2 K$. Since this holds for all initial wealth levels it would also hold for the initial wealth $w+200$; hence we know that a wealth increase from $w+200$ to $w+400$ implies a $U$ increase of less then $K / 2$, and that a wealth increase from $w+200(r-1)$ to $w+200 r$, where $r$ is an arbitrary positive integer larger than 1 , implies a utility increase of less than $(K / 2)^{r-1}$. Hence, the utility change for a wealth increase from $w$ to $w+200 r$ is less than $\sum_{i=0}^{r-1} K / 2^{i}=2\left(1-0.5^{r}\right) K$. Consequently, the expected utility change of a 50-50 lose $\$ 200 /$ gain $\$ 200 r$ gamble is less than $\left(1-0.5^{r}\right) K-K=-0.5^{r} K$. Thus, the expected utility change is negative irrespective of $r$, i.e. irrespective of the gain. (One can easily obtain less extreme versions by replacing the "for all wealth levels" with "for wealth levels up to $w+\Delta w$.") Moreover, by replacing $\$ 100$ with an arbitrary positive number $A$ in the above analysis, it follows more generally that, "If for all wealth levels an expected utility maximizing person turns down a 50-50 lose A/gain $2 A$ gamble, he would also turn down a 50-50 lose 2A/gain infinity gamble."
} 
that the individual would have made the same choice had he been substantially wealthier than what he actually is (see Rabin and Thaler and footnote 1 in the present paper). Largely based on the implications of this theorem, Rabin (2000a, b) and Rabin and Thaler (2001) argue more generally that EU theory cannot explain behavior based on small-stake gambles, and hence that we need some other theory; they suggest a combination of loss aversion and mental accounting.

However, Cox and Sadiraj (2006) question this conclusion in a recent paper. They show that for the small-stake risk aversion assumption of Rabin (2000), implausible large-stake risk aversion would not follow for the expected utility of income (EUI) model, where utility is expressed as a function of payoffs, in contrast to the expected utility of final wealth (EUW) model. ${ }^{2}$ Moreover, since the global small-stake risk aversion assumed by Rabin (2000) has no implication for the EUI model, it has no general implication for EU theory either. It is clear that Cox and Sadiraj have a valid and important point since EU theory is very general and builds on a set of axioms that do not preclude that utility may depend on wealth, income, experimental payoffs, or almost any state variable. ${ }^{3}$

In the light of the findings by Cox and Sadiraj, one may be inclined to conclude that what has become known as the Rabin critique is overstated. Perhaps applied economists interested in measuring people's risk preferences or analyzing behavior based on existing estimates can ignore the Rabin critique and continue to interpret their results in terms of the concavity of universally valid utility functions? However, the results in this paper suggest that such a conclusion would be premature.

In applied economic analysis people often make decisions over time, deriving instantaneous utility based on their present consumption level. Under risk, the conventional

\footnotetext{
${ }^{2}$ Although it is clear from Rabin (2000 a, b) and Rabin and Thaler (2001, 2002) that they focus on the EUW model in their analyses, some of their statements may seem to imply (or at least have been interpreted to imply) a criticism of expected utility theory more generally. Cox and Sadiraj (2006) also consider a more general twoargument model where utility depends on both initial wealth and payoff.

${ }^{3}$ Samuelson (2005), Rubinstein (2006), and Harrison et al. (2007) have provided similar arguments.
} 
assumption is then that people maximize the expected present value of future instantaneous utility (e.g. Deaton 1992; Gollier 2001). We will denote this model the expected utility of consumption over time (EUCT) model, and take it as our point of departure. An obvious example is how to best invest retirement savings; see e.g. Gomes and Michaelides (2005). ${ }^{4}$ In the EUCT model, utility is expressed as a function of a flow variable (unlike the EUW model), i.e. consumption, and implies complete asset integration (as in the EUW model), meaning that the gains from a risky choice will be treated in exactly the same way as income or wealth obtained in any other way.

The main contributions of the present paper can be summarized as follows: First, the relations between the EUCT model, on the one hand, and the EUW and EUI models, on the other, are analyzed in Section 2. It is concluded that the EUCT model is essentially equivalent to the EUW model when the wealth measure in the EUW model consists of the present value of all future consumption or income. In addition, it is shown that the functional form of the instantaneous utility function, which is expressed as a function of current consumption, carries over in a straightforward way to a utility function that is expressed as a function of the present value of all future incomes, if and only if the instantaneous utility function belongs to the class of functions characterized by hyperbolic absolute risk aversion (HARA), which is a flexible functional form that includes CRRA and CARA functions as special cases (Merton, 1971).

Second, by using data from a careful experimental study by Holt and Laury (2002), Section 3 analyzes whether observed behavior in small- and intermediate-stake size risk experiments can be reconciled by the EUCT model. The answer is negative. The calculated implicit risk aversion parameters are found to be unreasonably large, and therefore can not

\footnotetext{
${ }^{4}$ Long-run environmental problems, such as the greenhouse effect, constitute another important example where both time and risk are crucial. It is also typically shown that the profitability of extensive abatement today depends critically on the discount rate chosen, which in turn depends strongly on the concavity of the instantaneous utility function; see e.g. Stern (2006) and Nordhaus (2007).
} 
constitute concavity measures of universally valid instantaneous utility functions. Moreover, strong implications are derived with respect to what these degrees of risk aversion would correspond to for long-term risky choices in terms of future income or consumption levels. For example, whether based on constant relative risk aversion (CRRA) or constant absolute risk aversion (CARA) preferences, a majority of the subjects from Holt and Laury would (given that they are EUCT maximizers) in the base case prefer an income level that with certainty would enable them to for the rest of their lifetime consume 36,000 USD annually, rather than a risky alternative where they with a $1 \%$ probability would be able to consume 35,990 USD annually and with a 99\% probability an infinite amount. This clearly seems implausible. Similar implausible results are then obtained also for broader class of HARA preferences. Section 4 generalizes and demonstrates that the main conclusions hold also under uncertain future incomes and for a time-inconsistent formulation.

Compared to the results based on the EUW model by Rabin (2000) and Rabin and Thaler (2001), the results here are less general in the sense that they depend on specific functional forms. On the other hand, the results here are less restrictive in the sense that they do not rely on any assumption that the choices would have been the same for all lifetime wealth levels, or for any higher lifetime wealth levels than the individuals currently have or expect to obtain. ${ }^{5}$ Section 5 concludes that the standard EUCT model appears inconsistent with available experimental small- and intermediate-stake data.

\section{The EUCT model}

The standard approach when dealing with intertemporal choices under uncertainty is to maximize the expected present value of future instantaneous utility (e.g. Deaton 1992; Gollier

\footnotetext{
${ }^{5}$ However, note that the choices for CARA preferences would have been the same for all lifetime wealth levels. When utility is CRRA, by contrast, we know that an individual that is indifferent between accepting a risky gamble would always accept it for higher wealth levels than the present one.
} 
2001). Let us start with the intertemporal consumption choice under certainty and in the next step take risky decisions into account.

\subsection{The intertemporal choice problem and HARA preferences}

Here an individual experiences the instantaneous utility $u\left(c_{t}\right)$ at time $t$ (from now), where $u$ is increasing and strictly concave. Assume that the individual will live for $T$ more years, and, as is standard, an additive and time consistent utility formulation such that the individual will maximize

$$
U=\int_{0}^{T} u\left(c_{t}\right) e^{-\rho t} d t
$$

where $\rho$ is the pure rate of time preference, sometimes denoted the utility discount rate. We will refer to $U$ as utility. Under certainty, $U$ is purely ordinal, so that any monotonic transformation of $U$ is permissible and hence constitutes an equally valid measure of utility. Under risk, however, each possible utility outcome $U^{i}$ must be interpreted in a cardinal sense, so that only affine transformations are permissible. The expected value of $U, E(U)$, is nevertheless still ordinal. ${ }^{6}$ The intertemporal budget constraint implies that the present value of future consumption equals the present value of future income, so that

$$
\int_{0}^{T} c_{t} e^{-r t} d t=\int_{0}^{T} y_{t} e^{-r t} d t \equiv Y
$$

where $r$ is the market interest rate. The associated Lagrangean can then be written as $\mathcal{L}=\int_{0}^{T} u\left(c_{t}\right) e^{-\rho t} d t+\lambda \int_{0}^{T}\left(y_{t}-c_{t}\right) e^{-r t} d t$, implying the corresponding first order conditions

\footnotetext{
${ }^{6}$ This means for example that under certainty, $\breve{U} \equiv \ln U$ is an equally valid measure of utility as $U$, in the sense that an individual that chooses a consumption path in order to maximize $\breve{U}$, will also maximize $U$. However, the only transformations of $u$ that leaves the optimal consumption path unaffected are affine transformations; hence $u$ is cardinal and unique only up to affine transformations. Under uncertainty, where we choose between different lotteries, we instead want to maximize $E U=\sum_{i} p^{i} U^{i}$. The optimal choice would then be unaffected by any monotonic transformation of $E U$, whereas only affine transformations of $U^{i}$, describing the utility in state $i$, leave the choice unchanged generally, and are hence permissible. Consequently, $U^{i}$ is cardinal.
} 


$$
u^{\prime}\left(c_{t}\right)=\lambda e^{(\rho-r) t}=u^{\prime}\left(c_{0}\right) e^{(\rho-r) t},
$$

which together with the budget restriction determine the optimal consumption path. ${ }^{7}$ Since the individual maximizes $U$ given a certain present value of lifetime income $Y$, we can alternatively write $U=V(Y)$, for a fixed interest rate.

We will subsequently analyze implications of choices between small-stake lotteries with respect to what these choices would imply in terms of risk aversion measures when people are EUCT maximizers, and also what they would imply in terms of large-stake choices. In doing so, we would like to know the relationship between the instantaneous utility function and our measure of utility as expressed as a function of $Y$. More specifically, we would like to know under which conditions the functional form carries over from $u_{t}(\cdot)$ to $V(\cdot)$. For example, if $u_{t}(\cdot)$ is CRRA, can we then know that also $V(\cdot)$ is CRRA? If this is the case (and it turns out that it is), it simplifies the analysis largely, since it is then straight forward to reduce the dynamic problem to a static analogue and work with the $V$-function instead of the $u$-function. We will start by considering a more general result on the relation between $u_{t}(\cdot)$ to $V(\cdot)$, followed by a more specific result which is straight forward to apply in the subsequent numerical analysis:

Proposition 1. The functional form of $u$ carries over to $V$, in the sense that we can either write utility as $U=V(Y)$ or as $\hat{U}=u\left(c_{t}^{*}(Y)\right)$, where $\hat{U}$ is an affine transformation of $U$, if and only if any of the following equivalent conditions are fulfilled:

i. The optimal consumption in period $t$ can be written as an affine function of $Y$, such that $c_{t}^{*}=a_{t}(r)+b_{t}(r) Y$, where $a_{t}(r)$ and $b_{t}(r)$ may depend on $t$ and $r$, but are independent of $Y$.

ii. The instantaneous utility function $u$ is HARA, such that $u_{t}=\frac{\left(\alpha+\beta c_{t}\right)^{(\beta-1) / \beta}}{\beta-1}$.

\footnotetext{
${ }^{7}$ For example, when $\rho=r$ it follows that $u^{\prime}\left(c_{t}\right)=\lambda$, implying that also $c_{t}$ is constant over time. Intuitively, people want to smooth their consumption over their life cycle in order to equalize their marginal instantaneous utility of income, which is a standard result in dynamic consumption theory (e.g. Hall, 1978).
} 
Proof: see Appendix. Note that since $\hat{U}$ is an affine transformation of $U$, it is by definition an equally valid measure of utility. This means that as long as we know that the consumption path is optimal, the instantaneous utility in any point in time (e.g. at present or ten years from now) is an equally valid measure of utility, i.e. of the present value of the instantaneous utility over the whole lifetime period. Moreover, since $\hat{U}$ is an affine transformation of $U$, and not just a monotonic transformation, it follows that for HARA preferences $\hat{U}$ is also a valid measure of von Neumann-Morgenstern utility under risk. For our purposes, an even more useful result follows directly from Proposition 1, expressed in terms of the annuity $c^{0} \equiv Y / S$, where $S \equiv \frac{1-e^{-r T}}{r}$ is the annuity factor: ${ }^{8}$

Proposition 2. If the instantaneous utility function is $H A R A$, such that $u_{t}=\frac{\left(\alpha+\beta c_{t}\right)^{(\beta-1) / \beta}}{\beta-1}$, it follows that an affine transformation of $U, \tilde{U}$, is also HARA such that $\tilde{U}=\frac{\left(\alpha+\beta c^{0}\right)^{(\beta-1) / \beta}}{\beta-1}$.

Proof: see Appendix. Note first again that since $\tilde{U}$ is an affine transformation of $U, \tilde{U}$ is both a valid measure of utility under certainty, and a valid measure of von Neumann-Morgenstern utility under risk. This result will be used repeatedly in Section 3. Note also that Proposition 2 holds whether a constant consumption path is optimal or not, i.e. whether $\rho=r$ or not.

It is easy to verify that the Arrow-Pratt coefficient of absolute risk aversion, defined based on the instantaneous utility function, is in the HARA case given by $A_{t} \equiv-\frac{u_{t}{ }^{\prime}}{u_{t}{ }^{\prime}}=\frac{1}{\alpha+\beta c_{t}}$, and that the corresponding coefficient of relative risk aversion is given by $R_{t} \equiv-\frac{u_{t}{ }^{\prime \prime}}{u_{t}{ }^{\prime}} c_{t}=\frac{c_{t}}{\alpha+\beta c_{t}}$. It follows that the instantaneous utility function is characterized by

\footnotetext{
${ }^{8}$ Thus, an individual could exactly afford the constant consumption level $c^{0}$ for the rest of his lifetime.
} 
CRRA for the special case when $\alpha=0$, implying that $u_{t}=\frac{c_{t}^{(\beta-1) / \beta}}{\beta-1}=\frac{c_{t}^{1-R}}{1-R}$, where $R=1 / \beta$, and hence that we can write $\tilde{U}=\frac{\left(c^{o}\right)^{1-R}}{1-R}$. Similarly, $u$ converges towards CARA when $\beta$ approaches 0 , so $u_{t}=-\alpha e^{-c_{t} / \alpha}=-\frac{e^{-A c_{t}}}{A}$, where $A=1 / \alpha$, and we may write $\tilde{U}=-\frac{e^{-A c^{0}}}{A} .9$ These results will also be used in the numerical calculations in Section 3.

\subsection{Introducing risk}

Consider now a lottery with the income path $y_{t}^{i}$ for $t \geq 0$ with probability $p^{i}$, where the realized income path is revealed before the consumption path is chosen. Expected utility is then given by

$$
E U=\sum_{i=1}^{n}\left(\int_{0}^{T} u\left(c_{t}^{* i}\right) e^{-\rho t} d t\right) p^{i}=\sum_{i=1}^{n} V\left(Y^{i}+x^{i}\right) p^{i},
$$

where each element of the optimal consumption path $c_{t}^{*_{i}}$ will satisfy (3), and where $x^{i}$ is the lottery gain. Again, we see that the EUCT model is equivalent to the EUW model in the case where wealth is defined as the present value of all future incomes $Y$. Note that (17) holds generally, whereas in the case of HARA preferences we also have that the functional form carries over from $u_{t}(\cdot)$ to $V(\cdot)$. It is also noteworthy that Proposition 2 implies that the choice of an individual with HARA preferences in a choice between lotteries with different lifetime incomes, implying different feasible future constant consumption streams, is independent of the individual's time preference $\rho$.

Assuming that the potential gains $x$ (which can be positive or negative) from the lottery occur today, we can write expected utility as $E U=\sum_{i=1}^{n} V\left(Y+x^{i}\right) p^{i}$. According to the so-

\footnotetext{
${ }^{9}$ Note again that any affine transformations are permissible. Note also that while the parameter of relative risk aversion is dimension free and scale independent, the parameter of absolute risk aversion is not dimension free and can e.g. be expressed per dollar unit.
} 
called Arrow-Pratt approximation (see e.g. Gollier 2001, p. 22), for small risks the risk premium $\psi$ is approximately given by $\psi \approx A \frac{\operatorname{var}(x)}{2}$, so that $A \approx 2 \frac{\psi}{\operatorname{var}(x)}$ and hence $R \approx 2 \frac{\psi}{\operatorname{std}(x)} \frac{S c^{0}}{\operatorname{std}(x)}$, where $A \equiv-V^{\prime \prime} / V^{\prime}$ and $R \equiv-Y V^{\prime \prime} / V^{\prime}$ are the associated coefficients of absolute and relative risk aversion, respectively. The literature based on life cycle consumption behavior often refers to values of $R$ in the $0.5-3$ range. ${ }^{10}$ According to Kocherlakota (1996, 52), “A vast majority of economists believe that values above 10 (or, for that matter, above 5) imply highly implausible behavior.” The ratio between the present value of all future consumption and the standard deviation of the monetary outcome of a risk experiment is typically very large. This implies that the risk premium must be a tiny fraction of the standard deviation of the monetary outcome for the behavior in the risk experiment not to be described as "highly implausible" by the above quotation, which will be illustrated further in the next section.

\section{Numerical illustration based on data from Holt and Laury (2002)}

There are many suitable experimental studies that could be used to illustrate the implications of the above model, but let us here rely on the well-known and carefully undertaken study by Holt and Laury (2002), who elicited the risk preferences of (mainly) US university students by using real money experiments with different stake sizes. Each student made a number of pairwize choices between one less risky (Option I) and one more risky (Option II) gamble; see Table 1 for a relevant sub-set. Indifference between Option I and Option II then implies a certain degree of risk aversion, and the choices were ordered so that indifference between the options implies larger and larger risk aversion. By observing at what point a subject switched

\footnotetext{
${ }^{10}$ For example, Blundell et al. (1994) and Attanasio and Browning (1995) found, in most of their estimates, $R$ to be in the order of magnitude of 1 or slightly above. Vissing-Jørgensen (2002) found that $R$ differs between stockholders (approx. 2.5 to 3 ) and bond holders (approx. 1 to 1.2).
} 
to Option II, they obtained a risk aversion range in which the subject belongs. Holt and Laury used several different functional forms, including the flexible expo-power functional form that includes CRRA and CARA as special cases, but did not integrate the gains with other expected lifetime incomes, i.e. in line with the EUCT model.

\subsection{CARA and CRRA preferences}

In order to test the implications of the EUCT model with real data, let us first focus on the two most commonly used functional forms, CRRA and CARA, ${ }^{11}$ where the instantaneous utility function can hence be written as $u_{t}=c_{t}^{1-R} /(1-R)$ and $u_{t}=-e^{-A c_{t}}$, respectively. From Proposition 2 together with (4) we have that when an individual is indifferent between two lotteries, I and II, we have:

$$
\begin{aligned}
& \sum_{i=1}^{n} p_{i}^{\mathrm{I}}\left(c^{0}+x_{i}^{\mathrm{I}} / S\right)^{1-R}=\sum_{i=1}^{n} p_{i}^{\mathrm{II}}\left(c^{0}+x_{i}^{\mathrm{II}} / S\right)^{1-R}, \\
& \sum_{i=1}^{n} p_{i}^{\mathrm{I}} e^{-A x_{i}^{\mathrm{I}} / S}=\sum_{i=1}^{n} p_{i}^{\mathrm{II}} e^{-A x_{i}^{\mathrm{II}} / S} .
\end{aligned}
$$

From (5) and (6) we can easily solve numerically for $R$ and $A$.

Consider now for comparison the EUI model where the lotteries are evaluated in isolation, and hence independent of other incomes. The EUI model is therefore of course in general not consistent with EUCT. In the CRRA case we have:

$$
\sum_{i=1}^{n} p_{i}^{\mathrm{I}}\left(x_{i}^{\mathrm{I}}\right)^{1-R}=\sum_{i=1}^{n} p_{i}^{\mathrm{II}}\left(x_{i}^{\mathrm{II}}\right)^{1-R}
$$

Clearly, since $x$ is typically small compared to $S c^{0},(7)$ should generally result in a smaller $R$ than (5), when indifferent between the two lotteries. However, in the CARA case, where initial wealth does not affect choices, (6) still holds (corrected for the scale of $A$ ). The reason is of course that the expected utility change of a lottery is here independent on the initial

\footnotetext{
${ }^{11}$ Following convention, these names just reflect the functional form of the instantaneous utility function. What these functional forms imply in terms of actual choices under risk depends of course also about other assumptions of the model.
} 
wealth level, which is only true for CARA preference. Consequently, the EUI model is equivalent to the EUCT model for the CARA, and only the CARA, instantaneous utility function.

Consider first for comparison the result of the EUI model, where the experimental gains are evaluated independently of people's baseline income and wealth levels. It can be observed from Table 1 that, based on the CARA preferences as expressed in (6), the median parameter of absolute risk aversion $A$ is between 0.101 and 0.299 based on the low-stake lottery, and between 0.015 and 0.026 based on the high-stake lottery. Based on CRRA preferences, the median parameter of relative risk aversion $R$ is calculated from (7) to be between 0.146 and 0.411 based on the low-stake lottery, and between 0.411 and 0.676 based on the high-stake lottery.

Consider now the conventional EUCT model. In the CARA case, $A$ of course remains the same, since with CARA preferences the choice between risky options are independent of initial wealth; cf. e.g. Rabin and Weizsäcker (2007). Since the parameter estimates differ largely between the high- and low-stake lotteries, this suggests that CARA does not constitute a good approximation of subject preferences. However, the main concern here is whether the orders of magnitude constitute reasonable reflections of globally valid instantaneous utility functions. In the CRRA case, we clearly need estimates of $S$ and $c^{0}$ in order to solve for $R$ in (5). Let us therefore assume that the subjects are 20 years old, that they expect to live until they are 80 (i.e. that they have 60 years left), that the real market interest rate is $5 \%$ annually, and that they quite pessimistically will earn future incomes that will enable them to consume $c^{0}=10,000$ USD per year (at today’s price level). For example, the second high payoff lottery in the Holt and Laury experiment corresponds then to a lottery between the present values of future incomes, such that the subjects in option $I$ can afford a constant annual consumption of 
10002.1048 USD with probability 0.6 and 10001.6839 USD with probability 0.4 , and in option II 10004.0518 USD with probability 0.6 and 10000.10524 USD with probability 0.4.

As can be seen in Table 1, the median $R$ is now larger than 19,000 based on the lowstake lottery, and larger than 2,800 based on the high-stake lottery. These are clearly values way above what is generally considered to be plausible, i.e. values in the range of 0.5 to 3 , or in any case considerably smaller than $10 .^{12}$ Note that we have made no assumption regarding the pure rate of time preference $\rho$, and all results are independent of whether the students actually would prefer to have a future increasing or decreasing consumption path over time. If the future annual consumption of the subjects would be larger than 10,000 USD, then the implicit parameters of relative risk aversion would of course be even larger.

However, one may also believe that students have liquidity constraints and hence face a larger real interest rate than others. Let us therefore make the extreme assumptions of an annual real interest rate of 500\% (instead of 5\%). Solving for $R$ in (5) nevertheless again reveals absurdly large values, as the last column of Table 1 shows.

$<<$ Table 1 about here $>>$

Thus, we have seen that the choices in Holt and Laury imply absurdly large risk aversion coefficients if based on CRRA preferences, whereas the coefficients are identical between the EUI model and the EUCT model in the case of CARA preferences.

However. since $A$ is not dimension free, it may be difficult to have a good intuition about what a reasonable range of $A$ is. One perhaps tempting interpretation could be that the EUCT model works perfectly fine, but that people have CARA preferences (or similar) rather than CRRA preferences. However, even if one is willing to ignore the $A$ discrepancies between the small- and large-stake experiments, this is not a plausible conclusion. To see this, consider the following gamble: In a safe alternative the individual would obtain the present

\footnotetext{
${ }^{12}$ Independent from this study, Schechter (2007) also obtained absurdly large parameters of relative risk aversion in a risk experiments based on a sample in rural Paraguay.
} 
value of all future income equal to 5 million USD. In a risky alternative, the individual would instead with the probability of $1 \%$ obtain 4.9999 million USD, and with $99 \%$ obtain an infinite amount. Presumably, most people would prefer the risky alternative. However, an individual with $A=0.101$ would actually prefer the safe alternative. ${ }^{13}$ Hence, $A=0.101$ is indeed unreasonably large. We will next more systematically look into the implications of the choices in the Holt and Laury lotteries, for implied choices in large stake lotteries expressed in terms of future consumption possibilities.

Consider the choice between a safe and a risky option concerning a subject's future income. In the safe option he will with certainty for the rest of his life earn an amount corresponding to a constant annual consumption of $c^{S}$ per year. In the risky option he will with probability $p$ obtain the high future income level that corresponds to a constant annual consumption level of $c^{H}$, and with probability $1-p$ a low future income corresponding to the constant annual consumption level $c^{L}$. We can then solve for $c^{L}$ from (5) and (6) for the CRRA and the CARA cases as follows:

$$
\begin{aligned}
& c^{L}=\left(\frac{\left(c^{S}\right)^{1-R}-p\left(c^{H}\right)^{1-R}}{1-p}\right)^{\frac{1}{1-R}}, \\
& c^{L}=\frac{1}{A} \ln \left(\frac{1-p}{e^{-A c^{S}}-p e^{-A c^{H}}}\right) .
\end{aligned}
$$

In the special case where the "lucky" outcome implies an infinite consumption level, and where $R>1$ and $A>0$, (8) and (9) reduce to:

$$
\begin{aligned}
& c^{L}=(1-p)^{-1 /(1-R)} c^{S}, \\
& c^{L}=c^{S}+\frac{1}{A} \ln (1-p) .
\end{aligned}
$$

$<<$ Table 2 about here $>>$

\footnotetext{
${ }^{13}$ This is because $-e^{-0.101 \cdot 5 \cdot 10^{6}}>-0.01 e^{-0.101 \cdot 4.9999 \cdot 10^{6}}-0.99 \cdot 0$.
} 
Table 2 illustrates the case where the lucky consumption level is infinite, and where moreover the probability of a lucky outcome is as high as 99\%. Consider first the CRRA case with a 5\% interest rate. The first line of Table 2 reveals that indifference between the safe and the risky option implies the same $R$ as indifference between Option I and Option II in Table 1. Consequently, if people's behavior can be described by the EUCT model with CRRA preferences, the same fraction (66\%) would prefer the less risky option. This means that $66 \%$ of the subjects in Holt and Laury would actually prefer being able to consume 36,000 USD annually with certainty rather than being able to consume an infinite amount with a 99\% probability and 35,991 USD annually with a 1\% probability. If we instead draw on the results from the high payoff lottery in Holt and Laury, the results become less extreme, although only slightly. Indeed, as shown from the fifth line, as many as $62 \%$ would prefer the safe option (36,000 USD annually) before a risky one with a $1 \%$ probability of being able to consume 35,942 USD per year and a 99\% probability of gaining infinite consumption. If we consider the extreme case of 500\% interest per year, the implied choices are still absurd. Moreover, as observed in the third and fourth column of Table 2, when considering CARA (instead of CRRA) preferences the results are consistently even more extreme. ${ }^{14}$

\subsection{More general HARA preferences}

While HARA is the mostly used flexible functional form of the utility function, the second most used is the so-called Expo-power utility function (Saha, 1993). Both of these flexible forms include CRRA and CARA as special cases. However, since the Expo-power function has some unattractive characteristics, in particular in regions of extreme risk aversion, we will here focus on the HARA function. Still, we will briefly describe some features of the Expopower function, and how it in principle can be used, in the Appendix.

\footnotetext{
${ }^{14}$ An important reason for this is the pessimistic assumption regarding the subjects' future income that underlies the $R$ estimates in Table 1 .
} 
The HARA instantaneous utility function, $u_{t}=\frac{\left(\alpha+\beta c_{t}\right)^{(\beta-1) / \beta}}{\beta-1}$, implies decreasing absolute risk aversion $\left(\partial A_{t} / \partial c_{t}<0\right)$ for $\beta>0$, and increasing absolute risk aversion for $\beta<0$; we also observe decreasing relative risk aversion for $\alpha<0$ and increasing relative risk aversion for $\alpha>0$. It is also straightforward to see that this instantaneous utility function is globally concave as long as $-\alpha<\beta c_{t}$, and that both $A_{t}$ and $R_{t}$ are everywhere increasing in $\alpha$ and $\beta$. When an individual is indifferent between two lotteries, I and II, we have

$$
\sum_{i=1}^{n} p_{i}^{\mathrm{I}}\left(\alpha+\beta\left(c^{0}+x_{i}^{\mathrm{I}} / S\right)\right)^{(\beta-1) / \beta}=\sum_{i=1}^{n} p_{i}^{\mathrm{II}}\left(\alpha+\beta\left(c^{0}+x_{i}^{\mathrm{II}} / S\right)\right)^{(\beta-1) / \beta}
$$

From (12) we can solve for $\beta$ for a given value of $\alpha$, and vice versa, or solve for either $\alpha$ or $\beta$ for a specified relationship between them. It is convenient for presentational purposes to rewrite (12) for $\beta>0$ as

$$
\sum_{i=1}^{n} p_{i}^{\mathrm{I}}\left(\alpha / \beta+c^{0}+x_{i}^{\mathrm{I}} / S\right)^{(\beta-1) / \beta}=\sum_{i=1}^{n} p_{i}^{\mathrm{II}}\left(\alpha / \beta+c^{0}+x_{i}^{\mathrm{II}} / S\right)^{(\beta-1) / \beta},
$$

whereas we for $\beta<0$ instead have

$$
\sum_{i=1}^{n} p_{i}^{\mathrm{I}}\left(-\alpha / \beta-c^{0}-x_{i}^{\mathrm{I}} / S\right)^{(\beta-1) / \beta}=\sum_{i=1}^{n} p_{i}^{\mathrm{II}}\left(-\alpha / \beta-c^{0}-x_{i}^{\mathrm{II}} / S\right)^{(\beta-1) / \beta}
$$

Moreover, suppose now that $\alpha$ and $\beta$ have been identified based on a risk experiment, such as the one by Holt and Laury, for an individual. Let the same individual choose between a safe and a risky option regarding all future income levels, as in the previous case for CRRA and CARA preferences. Given indifference between the options we can solve for $c^{L}$ as follows:

$$
c^{L}=\left(\frac{1}{1-p}\left(\frac{\alpha}{\beta}+c^{S}\right)^{(\beta-1) / \beta}-\frac{p}{1-p}\left(\frac{\alpha}{\beta}+c^{H}\right)^{(\beta-1) / \beta}\right)^{\beta /(\beta-1)}-\frac{\alpha}{\beta}
$$

Let us now again focus on the extreme case where the high income outcome implies an infinite consumption level. For $\beta<1$, (14) then converges towards 


$$
c^{L}=(1-p)^{-\beta /(\beta-1)}\left(\frac{\alpha}{\beta}+c^{S}\right)-\frac{\alpha}{\beta} .
$$

In Table 3 below, we calculate $c^{L}$ for a very wide range of $\alpha / \beta \cdot{ }^{15}$ As observed, the implied choices are still absurd for almost all values of $\alpha / \beta$. Consider for example the case where $\alpha / \beta=-7000$. The number 35,977 in the fourth column should then be interpreted as follows: Assume that a student makes a choice between Option I and Option II in the first low payoff lottery choice described in Table 1, and that he has HARA preferences where the relation between $\alpha$ and $\beta$ is such that $\alpha=-7000 \beta$, where $\beta$ is a positive number. Based on the EUCT model with a 5\% annual interest rate, this implies that if he chooses Option I, he would prefer a future income stream allowing him to for the rest of his life consume 36,000 USD annually with certainty before a risky alternative where he with a 99\% probability would be able to consume an infinite amount and with a $1 \%$ probability would be able to consume 35,977 USD annually. This clearly seems implausible.

$<<$ Table 3 about here $>>$

The only exception occurs where $\alpha / \beta$ is very close to the negative of the baseline income level, which in our case occurs where $c^{0}=10,000$. Indeed, when $\alpha / \beta=-10,000$ we can write utility of a lottery outcome at state $i$ as $U^{i}=-0.17\left(-10,000+c^{0}+x^{i}\right)^{-5.85}=-0.17\left(x^{i}\right)^{-5.85}$, where $x^{i}$ here represent the possible constant consumption level on top of $c^{0}$. Hence, this function is equivalent to the CRRA EUI model at this value of $c^{0}$. This also means that the coefficient of the relative risk aversion

\footnotetext{
${ }^{15}$ Note that for the instantaneous utility function to be defined we must for $\beta>0$, i.e. where we have decreasing absolute risk aversion, have that $k>-\left(c^{0}+x_{i} / S\right)$ for all $x_{i}$. In the lottery about future wages we must then have that $k>-c^{L}$. When $\beta<0$, i.e. where we have increasing absolute risk aversion, we must have that $k<-\left(c^{0}+x_{i} / S\right)$. This means that the instantaneous utility function in this range is not defined for a sufficiently large consumption level. In our future wage lottery we must then have that $k<-c^{H}$. In order to still illustrate this (rather unrealistic) range of the HARA utility function, we choose $c^{H}=100000$ here.
} 
would be the same as for the EUI case, reported in Table 1 . Hence, we do not obtain the absurd choices in the example of future wages here. However, as shown below, we will still obtain unreasonable large stake choices close to the baseline consumption level.

So far we have drawn implications based on a single pair wise choice based on either the low payoff or high payoff lotteries of Holt and Laury. However, since we have two parameters in the HARA case we can actually estimate the parameters consistent with being indifferent in the first low-payoff pairwise lottery choice as well as the second high-payoff pairwise lottery choice. When doing so we obtain parameter values that are rather close to the case described above. Indeed, for the case where $r=5 \%$ annually, we can write utility as $U^{i}=-0.92\left(-9999.84+c^{0}+x^{i}\right)^{-1.09}=-0.92\left(0.16+x^{i}\right)^{-1.09}$. Here too, there are no extreme risk averse choices with respect to the above thought experiment of future wages. The reason is that in order to match indifference in both the first low-payoff pairwise choice and the second high-payoff pairwise choice of Holt and Laury, the utility function has to have an extreme curvature in this region. This, in turn, implies that the local risk aversion for small changes around $c^{0}=10,000$ will be extremely large, whereas it will decrease rapidly for larger levels. For example, the relative risk aversion at the benchmark consumption level $c^{0}=10,000$ is here equal to $R=\frac{c / \beta}{\alpha / \beta+c}=\frac{10,000 \cdot 0.479}{-9999.84+10,000}=29937.5$, whereas at the consumption level 36,000 we have $R=\frac{36,000 \cdot 0.479}{-9999.84+36,000}=0.18$. This implies that we will obtain absurd large stake risk aversion here too, but in another interval, namely close to the benchmark consumption level. Indeed, with these preferences an individual would prefer a safe option with a future income corresponding to a constant consumption level of 10,000 per year, instead of a risky option where he with $99.99 \%$ probability would obtain an infinite amount 
and with a $0.01 \%$ probability would obtain an amount corresponding to 9999.84 per year (since utility converges to minus infinity at this level).

Thus, we observe absurd large stake implications based on the choice behavior in the Holt and Laury experiments for all HARA utility functions consistent with either the behavior in the small stakes experiment, the large stake experiment, or both. Overall, it can then be concluded that the major conclusions about absurd large stake implications based on the choice behavior in the Holt and Laury experiment hold much more generally than for CRRA and CARA preferences.

\section{Generalizations}

In Section 3 we found that the EUCT model does not seem to be consistent with the experimental data provided by Holt and Laury (2002), and that absurd conclusions follow also when based on pessimistic forecasts regarding future income and with an extremely high interest rate. In this section we provide further generalizations. First we consider a setting where we take future income uncertainty into account. Then we consider possible self-control problems in terms of time-inconsistent present-bias preferences. As will be shown, the main findings are robust to these generalizations.

\subsection{Uncertainty in future income}

So far we have assumed perfect foresight, i.e. where future income is known, which can be questioned. Let us therefore now assume that future income is uncertain. Let us moreover assume that the true future income will be revealed only once, directly after the experimental outcome is observed. In this way, the impact of the uncertainty is maximized, since this makes it impossible to pool any future income risks. An individual would then maximize

$\int_{0}^{T} u\left(c_{t}\right) e^{-\rho t} d t$ as before, but this time subject to the budget constraint that $\int_{0}^{T} c_{t} e^{-r t} d t=\tilde{Y}$, 
where $\tilde{Y}$ is a stochastic variable representing the present value of all future income. The first order conditions are the same as before and given by (3), and expected utility is now given by

$$
E U=\sum_{i=1}^{n} \int_{Y^{\min }}^{Y^{\max }} p^{i} V\left(\tilde{Y}+x^{i}\right) f(\tilde{Y}) d \tilde{Y}
$$

Moreover, let the distribution of the future income per time unit be uniform, implying a uniform distribution also of $c^{0}$ (since $c^{0}=Y / S$ ). Besides facilitating simple expressions, this also implies thick "tails" in order to get large effects from the uncertainty of present risky choices. Combining this with HARA preferences, cf. eq. (12), we obtain

$$
\begin{aligned}
& E U=\sum_{i} \int_{c^{\min }}^{c^{\max }} p_{i}\left(\alpha+\beta\left(c^{0}+x_{i} / S\right)\right)^{(\beta-1) / \beta} \frac{\bar{c}}{c^{\max }-c^{\min }} d c^{0} \\
& =\frac{\bar{c}}{c^{\max }-c^{\min }} \frac{1}{(2 \beta-1)} \sum_{i} p_{i}\left[\left(\alpha+\beta\left(c^{0}+x_{i} / S\right)\right)^{(2 \beta-1) / \beta}\right]_{c^{\min } c^{\max }}, \\
& =\theta \sum_{i} p_{i}\left(\left(\alpha+\beta\left(c^{\max }+x_{i} / S\right)\right)^{(2 \beta-1) / \beta}-\left(\alpha+\beta\left(c^{\min }+x_{i} / S\right)\right)^{(2 \beta-1) / \beta}\right)
\end{aligned}
$$

where $\theta \equiv \frac{\bar{c}}{c^{\max }-c^{\min }} \frac{1}{(2 \beta-1)}$ is a constant. Indifference between the two lotteries I and II then implies that

$$
\begin{aligned}
& \sum_{i} p_{i}^{\mathrm{I}}\left(\left(\alpha+\beta\left(c^{\max }+x_{i}^{\mathrm{I}} / S\right)\right)^{(2 \beta-1) / \beta}-\left(\alpha+\beta\left(c^{\min }+x_{i}^{\mathrm{I}} / S\right)\right)^{(2 \beta-1) / \beta}\right) \\
& =\sum_{i} p_{i}^{\mathrm{II}}\left(\left(\alpha+\beta\left(c^{\max }+x_{i}^{\mathrm{II}} / S\right)\right)^{(2 \beta-1) / \beta}-\left(\alpha+\beta\left(c^{\min }+x_{i}^{\mathrm{II}} / S\right)\right)^{(2 \beta-1) / \beta}\right)
\end{aligned} .
$$

In order to obtain some numerical estimates, let us make the extreme assumptions that $c^{\min }=1000$ USD and $c^{\max }=19,000$ per year, so that the expected future income is the same as before (i.e. equally pessimistic). However, numerical calculations of the kind provided in Table 1 reveal in the CRRA case that the implied parameters of relative risk aversion are still absurdly large, although about a factor ten smaller than in the base case. The median values are between 1,926 and 5,675 and between 285 and 492 in the small- and large-stake lotteries, respectively, with a 5\% annual interest rate. The correspondingly implied choices as reported 
in Table 2 would of course then also be extreme. Consequently, the absurd results are not driven by our deterministic future income assumptions.

\subsection{Time inconsistent preferences}

So far, we have made the standard assumption that people have time-consistent intertemporal preferences. However, there is much empirical evidence that people in fact often do not make intertemporal choices in a time-consistent way (Frederick et al., 2002). Here we therefore generalize the model in order to take time inconsistency into account, by means of a present bias model similar to the one used in the seminal work by Laibson (1997), and many others. In our continuous time framework, the individual would then at each time period $t$ maximize

$$
U_{t}=\sigma u\left(c_{t}\right) d t+\int_{t+d t}^{T} u\left(c_{\tau}\right) e^{-\rho(\tau-t)} d \tau
$$

where $\sigma$ is the ratio between the weight attached to the present consumption and that for the near future, whereas the relative weights given to future time periods are given by the constant discount rate as in the standard exponential discounting case. In order to focus on the most extreme outcomes, we will solely focus on "naïve” individuals that will not today take into account that they will be time inconsistent also tomorrow; cf. O’Donoghue and Rabin (1999). The Lagrangean associated with the maximization at time $t$ can then be written

$$
\mathcal{L}=\sigma u\left(c_{t}\right) d t+\int_{t+d t}^{T} u\left(c_{\tau}\right) e^{-\rho(\tau-t)} d \tau+\lambda\left(Y_{t}-e^{r t} \int_{t}^{T} c_{\tau} e^{-r \tau} d \tau\right)
$$

where $Y_{t}=e^{r t}\left(Y-\int_{0}^{t} c_{s} e^{-r s} d s\right)$ is the present value of lifetime income minus the present value of consumption until time $t$, implying the corresponding first order condition for consumption at time $t$

$$
\sigma u^{\prime}\left(c_{t}\right)=\lambda,
$$


and for all future time after $t$

$$
u^{\prime}\left(c_{t+\tau}\right)=\lambda e^{-(r-\rho) \tau} .
$$

Consequently, the intertemporal marginal rate of substitution between two future time periods is given by $u^{\prime}\left(c_{t+\tau_{i}}\right) / u^{\prime}\left(c_{t+\tau_{j}}\right)=e^{-(r-\rho)\left(\tau_{i}-\tau_{j}\right)}$ as in the time consistent case, whereas the corresponding intertemporal marginal rate of substitution between the present and any future time period is given by $u^{\prime}\left(c_{t}\right) / u^{\prime}\left(c_{t+\tau}\right)=\sigma e^{(r-\rho) \tau}$.

Consider for example the case where the instantaneous utility function is characterized by CRRA, so that $u\left(c_{t}\right)=\frac{c_{t}^{1-R}}{1-R}$. Then it follows that $c_{0}=\sigma^{1 / R} c_{0}^{t c}=\sigma^{1 / R} \frac{\eta}{1-e^{-\eta T}} Y$, where $c_{0}^{t c}$ is the time consistent consumption at time 0, i.e. the consumption an individual would have if $\sigma=1$, and where $\eta=(r R+\rho-r) / R$. Similarly, $c_{t}=\sigma^{1 / R} c_{t}^{t c}$, where $c_{t}^{t c}$ is the consumption chosen by an individual who has been time inconsistent up to time $t$ but who will be time consistent from then on. Thus, $c_{t}=\frac{\sigma^{1 / R} \eta}{1-e^{-\eta(T-t)}} Y_{t}$, where again $Y_{t}$ is the present value of lifetime income minus the present value of consumption until time $t$. Then we have

$$
c_{t}=\frac{\sigma^{1 / R} \eta}{1-e^{-\eta(T-t)}} e^{r t}\left(Y-\int_{0}^{t} c_{s} e^{-r s} d s\right) .
$$

For example, when preferences are logarithmic $(R=1), \sigma=1.1$ and $\rho=r$, so that a time consistent individual would prefer a constant consumption level, it follows that an individual will always consume $10 \%$ more per time unit than he can afford to consume per time unit during the rest of his lifetime. By differentiating the integral equation (or more precisely the Volterra integral equation of the second kind) in (22) by $t$, and then substituting in (22), we obtain the following separable differential equation 


$$
\begin{aligned}
& \frac{d c_{t}}{d t}=-\frac{\sigma^{1 / R} \eta}{1-e^{-\eta(T-t)}} e^{r t} c_{t} e^{-r t}+\left(Y-\int_{0}^{t} c_{s} e^{-r s} d s\right)\left(\frac{\sigma^{1 / R} \eta^{2}}{\left(1-e^{-\eta(T-t)}\right)^{2}} e^{-\eta(T-t)+r t}+\frac{\sigma^{1 / R} \eta}{1-e^{-\eta(T-t)}} r e^{r t}\right) . \\
& =\frac{(\eta-r) e^{-\eta(T-t)}+r-\sigma^{1 / R} \eta}{1-e^{-\eta(T-t)}} c_{t}
\end{aligned}
$$

It is straightforward to solve (23), for $\eta>0$, as

$$
\int \frac{d c_{t}}{c_{t}}=\int \frac{(\eta-r) e^{-\eta(T-t)}+r-\eta \sigma^{1 / R}}{1-e^{-\eta(T-t)}} d t
$$

implying

$$
\ln c_{t}=\left(r-\eta \sigma^{1 / R}\right) t+\left(\sigma^{1 / R}-1\right) \ln \left(e^{\eta T}-e^{\eta t}\right)+K,
$$

or

$$
C_{t}=e^{\left(r-\eta \sigma^{1 / R}\right) t}\left(e^{\eta T}-e^{\eta t}\right)^{\left(\sigma^{1 / R}-1\right)} e^{K}
$$

where $K$ is the constant of integration. $K$ can be identified by the initial condition $c_{0}=\frac{\eta \sigma^{1 / R}}{1-e^{-\eta T}} Y$, implying $e^{K}=\left(e^{\eta T}-1\right)^{-\left(\sigma^{1 / R}-1\right)} \frac{\eta \sigma^{1 / R}}{1-e^{-\eta T}} Y$, which substituted in (24) implies ${ }^{16}$

$$
C_{t}=\frac{\eta \sigma^{1 / R}}{1-e^{-\eta T}} \frac{\left(e^{\eta T}-e^{\eta t}\right)^{\left(\sigma^{1 / R}-1\right)}}{\left(e^{\eta T}-1\right)^{\left(\sigma^{1 / R}-1\right)}} e^{\left(r-\eta \sigma^{1 / R}\right) t} Y
$$

A person who repeatedly and consistently behaves in this way will then end up with much lower consumption when old even for a $\sigma$ that is relatively close to one. Indeed, in this case it follows that the consumption will converge towards zero at the end of life when $\sigma>1$, i.e. irrespective of $\rho$ and $r$, since $\left(e^{\eta T}-e^{\eta t}\right)^{\left(\sigma^{1 / R}-1\right)}$ will then converge towards zero. ${ }^{17}$ We can ${ }^{16}$ For $\eta<0$ we obtain instead the optimal consumption path as follows: $c_{t}=-\frac{\eta \sigma^{1 / R} e^{(r-\eta) t}\left(e^{-\eta T}-e^{-\eta t}\right)^{\left(\sigma^{1 / R}-1\right)}}{\left(e^{-\eta T}-1\right)^{\sigma^{1 / R}}} Y$. Similarly, for $\eta=0$ we get $c_{t}=-\sigma^{1 / R} e^{r t} T^{1-\sigma^{1 / R}}(T-t)^{-2+\sigma^{1 / R}} Y$. The same reasoning can be used for the more general case of HARA preferences, although the integral and differential equations naturally become substantially more complicated in this case (results are available from the author upon request).

${ }^{17}$ See Diamond and Köszegi (2003) for a discrete time application to pension savings. 
then obtain non-monotonic consumption paths, i.e. paths that are initially increasing up to a certain point in time and thereafter decreasing, when $\rho<r$. For example, a 20 year old individual who expects to live until the age of 80, with $R=1, \sigma=1.3, \rho=0.03$, and where $r=0.05$, would reach a peak consumption at about the age of 60 .

Let us next consider the direct implications of time-inconsistent preferences for risky choices by again writing the expected utility when an individual faces a lottery with $n$ different prices. Letting $G_{t}=\frac{\eta \sigma^{1 / R}}{1-e^{-\eta T}} \frac{\left(e^{\eta T}-e^{\eta t}\right)^{\left(\sigma^{1 / R}-1\right)}}{\left(e^{\eta T}-1\right)^{\left(\sigma^{1 / R}-1\right)}} e^{\left(r-\eta \sigma^{1 / R}\right) t}$ we can write (25) as $c_{t}=G_{t} Y$, so that we may write expected utility as

$$
\begin{aligned}
& E U=\sum_{i=1}^{n}\left(\sigma \frac{\left(G_{0}\left(Y+x_{i}\right)\right)^{1-R}}{1-R} d t+\int_{0}^{T} \frac{\left(G_{t}\left(Y+x_{i}\right)\right)^{1-R}}{1-R} e^{-\rho t} d t\right) p^{i} \\
& =\sum_{i=1}^{n} \frac{\left(Y+x_{i}\right)^{1-R}}{1-R}\left(\sigma \frac{G_{0}^{1-R}}{1-R} d t+\int_{0}^{T} \frac{G_{t}^{1-R}}{1-R} e^{-\rho t} d t\right) p^{i} \\
& =\Upsilon \sum_{i=1}^{n} p_{i} \frac{\left(Y+x_{i}\right)^{1-R}}{1-R}=\Upsilon^{\prime} \sum_{i=1}^{n} p_{i} \frac{\left(c^{0}+x_{i} / S\right)^{1-R}}{1-R}
\end{aligned}
$$

where $\Upsilon$ and $\Upsilon$ 'are arbitrary positive constants. Thus, these final expressions look exactly as in the time consistent case in eq. (12), i.e. they do not depend at all on $\sigma$ ! Hence, although time inconsistency in the form of present bias preferences can have very large implications on the consumption path and saving decisions, the implications for the behavior in risky choices are much smaller, and in our case none. Consequently, our standard, but perhaps unrealistic, assumption about time consistency in our initial EUCT formulation is not what drives the absurd implications of the choices made in the Holt and Laury (2002) data.

\section{Discussion and Conclusion}

The explanatory power of EU theory has recently been discussed intensively (e.g. Rabin 2000a; Rabin and Thaler 2001; Cox and Sadiraj 2006; Rubinstein 2006). The present paper is 
concerned with the implications of this discussion for applied economics research under risk. In such research people often make decisions over time, where the standard model (here denoted the EUCT model) assumes that people maximize the expected present value of future instantaneous utility. The relations between the EUCT model and the EUW and EUI models, respectively, are analyzed. It is shown that the EUCT model is equivalent to the EUW model when wealth is measured by the present value of all future consumption or income, whereas the EUCT model is equivalent to the EUI model only for CARA preferences. Moreover, it is shown that the functional form of the instantaneous utility function, which is expressed as a function of current consumption, carries over in a straightforward way to a utility function that is expressed as a function of the present value of all future incomes, if and only if the instantaneous utility function belongs to the class of functions characterized by hyperbolic absolute risk aversion, which includes CRRA and CARA as special cases.

However, as argued by Palacios-Huerta and Serrano (2006), the important question is not whether the EU model, or in our case the EUCT model, is literally correct. We know that it is not. What is important for applied economics is the extent to which the model can provide a reasonable approximation of actual behavior. Here the implications of the EUCT model have therefore been investigated based on data from a careful risk experiment reported in Holt and Laury (2002). The result suggests that the EUCT model is ill-suited to explain experimental behavior in such small- and intermediate-stake gambles. The calculated implicit risk-aversion parameters are found to be unreasonably large, and therefore can not constitute concavity measures of universally valid instantaneous utility functions. For example, in the base case with CRRA preferences, making conservative or realistic assumptions regarding future wages, etc., the median coefficient of relative risk aversion is above 2,000 even based on the high-stake lottery choices, despite that most analysts seem to agree that $R$ should be in the 0.5-3 range, or at least not larger than 10 . 
Even more strikingly, the results also suggest unreasonable implications in terms of what these degrees of risk aversion would correspond to for long-term risky choices in terms of the subjects' future consumption levels. Whether based on CRRA or CARA preferences, most subjects from Holt and Laury would in the base case EUCT model prefer a certain income enabling them to for the rest of their lifetime consume 36,000 USD annually instead of a risky alternative where they with a $1 \%$ probability would be able to consume 35,990 USD annually and with a 99\% probability would be able to consume an infinite amount. Moreover, since the results in Holt and Laury (2002) are in no way unique, but are in line with most small and intermediate stake experimental risk studies, ${ }^{18}$ it can be concluded that the EUCT model appears inconsistent with observed experimental small- and intermediatestake data. The same applies to at least some kinds of actual consumption behavior, such as additional insurances for electronic equipment.

However, a caveat regarding the numerical results is in order. The numerical analysis here implicitly assumes that the choices reported in Holt and Laury are without errors, which is of course not the case in reality. Moreover, when extrapolating to implied choices at a much larger scale, as is done here, such errors will of course increase too, as pointed out by Cox and Harrison (2008). This means that the numerical results here should accordingly be taken with some grain of salt. Still, in order to escape the main conclusions, one has to assume that the choices in Holt and Laury are almost solely driven by random, which seems unlikely.

Consequently, we need another model to explain small- and intermediate-stake risk behavior. There are several suggestions well worth considering in the literature; see e.g. Rabin and Thaler (2001), Köbberling and Wakker (2005), Barberis et al. (2006), Cox et al. (2008), Heinemann (2008), and Rabin and Weizsäcker (2007). Although it is beyond the scope of the present study to discriminate between these and other models, two remarks appear clear based

\footnotetext{
${ }^{18}$ See e.g. Cox and Sadiraj (2008) for a discussion and analysis of other recent risk experiments.
} 
on the findings here: 1. Loss aversion cannot explain the choice behavior in Holt and Laury, and hence not the numerical findings in this paper, since all outcomes were in the gain domain (unlike the thought experiments by Rabin and Thaler). 2. It appears that a successful model should include the element that a decision-maker who faces multiple decisions in each case tend to make a decision with insufficient regard to the other decisions, and hence with insufficient regard to income or wealth from other sources. ${ }^{19}$

Finally, since repeated games also add payoffs over time, one may worry that repeated game theory would be in trouble too. However, as noted by an advisory editor, the results from this paper do not justify such a conclusion. An important difference is that in repeated game theory, utility in a certain period depends only on the choices made in that period while in the EUCT model an increased consumption reduces future utility. The extent by which people integrate the payoffs from a certain stage in a game with the payoffs from other stages, as well as with background lifetime wealth, is a separate and important issue that is left for future research.

\section{References}

Attanasio, O. P., Browning, M., 1995. Consumption over the life cycle and over the business cycle. American Economic Review 85, 1118-1137.

Barberis, N., Huang, M., Thaler, R.H., 2006. Individual Preferences, Monetary Gambles, and Stock Market Participation: A Case for Narrow Framing. American Economic Review 96, 1069-1090.

Blundell, R., Browning, M., Meghir, C., 1994, Consumer demand and the life-cycle allocation of household expenditures. Review of Economic Studies 61, 57-80.

Cox, J.C., Harrison, G.W., 2008. Risk Aversion in Experiments: An Introduction. Ch. 1 in J. C. Cox and G. W. Harrison (eds.), Risk Aversion in Experiments, Research in Experimental Economics, Vol. 12, Cornwall: Emerald JAI Press, pp. 1-7.

\footnotetext{
19 This and similar phenomena have different names in the literature, including decision isolation, mental accounting, narrow bracketing, narrow framing and partial asset integration.
} 
Cox, J.C., Sadiraj, V., 2006. Small- and large-stakes risk aversion: Implications of concavity calibration for decision theory. Games and Economic Behavior 56, 45-60.

Cox, J.C., Sadiraj, V., 2008. Risky Decisions in the Large and in the Small: Theory and Experiment, Ch. 2 in J. C. Cox and G. W. Harrison (eds.), Risk Aversion in Experiments, Research in Experimental Economics, Vol. 12, Cornwall: Emerald JAI Press, pp. 9-40.

Cox, J.C., Sadiraj, V., Vogt, B., Dasgupta, U., 2009. Is There a Plausible Theory for Risky Decisions? Updated version of Experimental Economics Center Working Paper 2008 04, Georgia State University.

Deaton, A., 1992. Understanding Consumption. Oxford: Oxford University Press.

Diamond, P., Köszegi, B., 2003. Quasi-hyperbolic discounting and retirement. Journal of Public Economics 87, 1839-1872.

Frederick, S., Loewenstein, G., O’Donoghue, T., 2002. Time discounting and time preference: a critical review. Journal of Economic Literature 40, 351-401.

Gollier, C., 2001. The Economics of Risk and Time, Boston: MIT Press.

Gomes F., Michaelides, A., 2005. Optimal Life-Cycle Asset Allocation: Understanding the Empirical Evidence. Journal of Finance 60, 869-904.

Hall, R.E., 1978. Stochastic implications of the life cycle/permanent income hypothesis; theory and evidence. Journal of Political Economy 86, 971-987.

Harrison, G.W., Lau, M.I., Rutström, E.E., 2007. Estimating Risk Attitudes in Denmark: A Field Experiment. Scandinavian Journal of Economics 109, 341-368.

Heinemann, F., 2008. Measuring risk aversion and the wealth effect, in: James C. Cox and Glenn W. Harrison (eds.), Risk Aversion in Experiments, Research in Experimental Economics, Vol. 12, Cornwall: Emerald JAI Press, pp. 293-313.

Holt, C.A., Laury, S.K., 2002. Risk aversion and incentive effects. American Economic Review 92, 1644-1655.

Kocherlakota, N., 1996. The Equity Premium: It's Still a Puzzle. Journal of Economic Literature 34, 42-71.

Köbberling, V., Wakker, P.P., 2005. An index of loss aversion. Journal of Economic Theory $122,119-131$.

Laibson, D., 1997. Golden Eggs and Hyperbolic Discounting. Quarterly Journal of Economics 112, 443-477.

Merton, R.C., 1971. Optimum Consumption and Portfolio Rules in a Continuous Time Model. Journal of Economic Theory 3, 373-413. 
Nordhaus, W., 2007. A Review of the Stern Review on the Economics of Climate Change. Journal of Economic Literature 45, 686-702.

O’Donoghue, T., Rabin, M., 1999. Doing it now or later. American Economic Review 89, 103-124.

Palacios-Huerta, I., Serrano, R., 2006, Rejecting Small Gambles under Expected Utility. Economics Letters 91, 250-259.

Rabin, M., 2000a. Risk aversion and expected utility theory: A calibration theorem. Econometrica 68, 1281-1292.

Rabin, M., 2000b. Diminishing marginal utility of wealth cannot explain risk aversion. In D. Kahneman and A. Tversky, editors, Choices, Values and Frames, Cambridge University Press.

Rabin, M., Thaler, R.H., 2001. Anomalies: risk aversion. Journal of Economic Perspectives 15, 219-232.

Rabin, M., Thaler, R.H., 2002. Response: (Anomalies: Risk Aversion). Journal of Economic Perspectives 16, 229-30.

Rabin, M., Weizsäcker, G., 2007. Narrow Bracketing and Dominated Choices, American Economic Review, forthcoming.

Rubinstein, A., 2006. Dilemmas of an economic theorist. Econometrica 74, 865-84.

Saha, A., 1993. Expo-power utility: A Flexible Form for Absolute and Relative Risk Aversion. American Journal of Agricyultural Economics 75, 905-913.

Samuelson, L., 2005. Foundations of Human Sociality: A Review Essay. Journal of Economic Literature 43, 488-497.

Schechter, L., 2007. Risk Aversion and Expected-Utility Theory: A Calibration Exercise. Journal of Risk and Uncertainy 35, 67-76.

Stern, N. 2007. The Economics of Climate Change: The Stern Review. Cambridge, UK, Cambridge University Press.

Tversky, A., Kahneman, D., 1981. The framing of decisions and the psychology of choice. Science 211, 453-458.

Vissing-Jørgensen, A., 2002, Limited Asset Market Participation and the Elasticity of Intertemporal Substitution. Journal of Political Economy 4, 825-853. 


\section{Appendix}

Proof of Proposition 1.

Let $c_{t}^{*}$ denote the optimal consumption at time $t$ and consider the welfare effect of a small lifetime income increase:

$$
\frac{d U}{d Y}=\frac{\partial V}{\partial Y}=\int_{0}^{T} u^{\prime}\left(c_{t}^{*}\right) \frac{\partial c_{t}^{*}}{\partial Y} e^{-\rho t} d t=u^{\prime}\left(c_{0}^{*}\right) \int_{0}^{T} \frac{\partial c_{t}^{*}}{\partial Y} e^{-r t} d t=u^{\prime}\left(c_{0}^{*}\right)
$$

where we have used (3) and $\int_{0}^{T} \frac{\partial c_{t}^{*}}{\partial Y} e^{-r t} d t=1$ from differentiating the budget restriction (2). Intuitively, the utility increase of consuming everything of a small income increase immediately is equally large as any other pattern of the consumption increase, as long as the initial consumption pattern is optimal. By combining (3) and (A1) we also have $\lambda=\partial V / \partial Y$. When $r$ is given we can write $U=V(Y)$, and from (3) we then have $\frac{d V}{d Y}=u^{\prime}\left(c_{t}^{*}(Y)\right) e^{(r-\rho) t}$ so

$$
V(Y)=e^{(r-\rho) t} \int u^{\prime}\left(c_{t}^{*}(Y)\right) d Y+\bar{K},
$$

where $\bar{K}$ is independent of $Y$. Now, since $\frac{d\left(u\left(c_{t}^{*}(Y)\right)\right)}{d y}=u^{\prime}\left(c_{t}^{*}(Y)\right) \frac{\partial c_{t}^{*}}{\partial Y}$ we have that $\int u^{\prime}\left(c_{t}^{*}(Y)\right) d y=\frac{u\left(c_{t}^{*}(Y)\right)}{\partial c_{t}^{*} / \partial Y}+\widehat{K}$, where $\hat{K}$ is independent of $Y$, if and only if $\frac{\partial c_{t}^{*}}{\partial Y}$ is independent of $Y$, i.e. when we can write

$$
c_{t}^{*}=a_{t}(r)+b_{t}(r) Y
$$

so that $\frac{\partial c_{t}^{*}}{\partial Y}=b_{t}(r)$. Hence, there is an affine relationship between $V$ and $u$ such that we may write

$$
U=V(Y)=K^{1} u\left(c_{t}^{*}(Y)\right)+K^{2},
$$


where $K^{1}$ and $K^{2}$ are independent of $Y$, if and only if (A3) holds. This implies in turn that we may write

$$
\hat{U}=u\left(c_{t}^{*}(Y)\right)
$$

where $\hat{U}$ is an affine transformation of $U$, and hence an equally valid measure of utility. This means that, when consumption is optimal, the instantaneous utility at any point in time is a measure of overall utility $U$.

Next we will consider the implications of affine optimal consumption functions for the underlying utility function, and show that $i$ implies ii. In doing so we will make use of implications due to changes in the interest rate, so we will write $U=V(Y, r)$ here. By differentiating (3) with respect to $Y$ we obtain

$$
u^{\prime \prime}\left(c_{t}\right) \frac{\partial c_{t}^{*}}{\partial Y}=u^{\prime \prime}\left(c_{t}\right) b_{t}(r)=\frac{\partial \lambda}{\partial Y} e^{(\rho-r) t}=\frac{\partial^{2} V}{\partial Y^{2}} e^{(\rho-r) t} .
$$

Similarly, differentiating (3) with respect to the interest rate $r$, we obtain

$$
u^{\prime \prime}\left(c_{t}\right) \frac{\partial c_{t}^{*}}{\partial r}=u^{\prime \prime}\left(c_{t}\right)\left(\frac{\partial a_{t}}{\partial r}+\frac{\partial b_{t}}{\partial r} Y\right)=\frac{\partial^{2} V}{\partial Y \partial r} e^{(\rho-r) t}-\frac{\partial V}{\partial r} t e^{(\rho-r) t} .
$$

By Youngs theorem we have $\frac{\partial^{2} V}{\partial Y \partial r}=\frac{\partial^{2} V}{\partial r \partial Y}$. Differentiating (1) with respect to $r$ implies

$$
\frac{\partial V}{\partial r}=\int_{0}^{T} u^{\prime}\left(c_{t}^{*}\right) \frac{\partial c_{t}^{*}}{\partial r} e^{-\rho t} d t=\frac{\partial V}{\partial Y} \int_{0}^{T}\left(\frac{\partial a_{t}}{\partial r}+\frac{\partial b_{t}}{\partial r} Y\right) e^{-r t} d t
$$

from which it follows that

$$
\frac{\partial^{2} V}{\partial r \partial Y}=\frac{\partial^{2} V}{\partial Y^{2}} \int_{0}^{T} \frac{\partial a_{t}}{\partial r} e^{-r t} d t+\frac{\partial^{2} V}{\partial Y^{2}} Y \int_{0}^{T} \frac{\partial b_{t}}{\partial r} e^{-r t} d t+\frac{\partial V}{\partial Y} \int_{0}^{T} \frac{\partial b_{t}}{\partial r} e^{-r t} d t
$$

where in the last line (3) and (A5) are used. Substituting (A7) and (A8) into (A6) and dividing by (A5) implies 


$$
\frac{\frac{\partial a_{t}}{\partial r}+\frac{\partial b_{t}}{\partial r} Y}{b_{t}(r)}=\int_{0}^{T} \frac{\partial a_{t}}{\partial r} e^{-r t} d t+Y \int_{0}^{T} \frac{\partial b_{t}}{\partial r} e^{-r t} d t-\frac{\frac{\partial V}{\partial Y}}{\frac{\partial^{2} V}{\partial Y^{2}}}\left(t-\int_{0}^{T} \frac{\partial b_{t}}{\partial r} e^{-r t} d t\right)
$$

so that

$$
-\frac{\frac{\partial^{2} V}{\partial Y^{2}}}{\frac{\partial V}{\partial Y}}=\frac{1}{k^{1}+k^{2} Y}
$$

where $k^{1}=\left(\int_{0}^{T} \frac{\partial a_{t}}{\partial r} e^{-r t} d t-\frac{\partial a_{t}}{\partial r} / b_{t}\right) /\left(t-\int_{0}^{T} \frac{\partial b_{t}}{\partial r} e^{-r t} d t\right)$ and

$$
k^{2}=\left(\int_{0}^{T} \frac{\partial b_{t}}{\partial r} e^{-r t} d t-\frac{\partial b_{t}}{\partial r} / b_{t}\right) /\left(t-\int_{0}^{T} \frac{\partial b_{t}}{\partial r} e^{-r t} d t\right) \cdot \cdot^{20}
$$

By definition, (A9) implies that $V$ belongs to the class of utility functions characterized by HARA in $Y$. And since $V$ is HARA in $Y$, we know from above that $u$ is also HARA in $c$ (that can also easily be verified by dividing (A5) by (3) when $V$ is HARA). What remains to be shown is that if $u$ is HARA in $c$ then the optimal consumption functions are affine functions of $Y$ and that $V$ is HARA in $Y$.

A HARA instantaneous utility function can be written as

$$
u\left(c_{t}\right)=\frac{\left(\alpha+\beta c_{t}\right)^{(\beta-1) / \beta}}{\beta-1} .
$$

By differentiating (A10) and substituting into (3), we get

$$
c_{t}=\frac{\lambda^{-\beta} e^{-\beta(\rho-r) t}-\alpha}{\beta}
$$

From the budget condition (2), we get $\lambda^{-\beta}=\frac{\beta((r(1-\beta)+\beta \rho))}{\left(1-e^{-(r(1-\beta)+\beta \rho) T}\right)}\left(Y+\frac{\alpha}{\beta r}\left(1-e^{-r T}\right)\right)$, so that the optimal consumption path is given by

\footnotetext{
${ }^{20}$ Note that $k^{1}$ and $k^{2}$ are constants, due to the fact that the left-hand-side of (A9) is time invariant. This of course has implications for the pattern of time dependency of $a_{t}$ and $b_{t}$, but these will not explore further here.
} 


$$
c_{t}^{*}=M e^{\beta(r-\rho) t}\left(Y+\frac{\alpha}{\beta} \frac{1-e^{-r T}}{r}\right)-\frac{\alpha}{\beta},
$$

where $M \equiv \frac{r(1-\beta)+\beta \rho}{1-e^{-(r(1-\beta)+\beta \rho) T}}$ is a constant; hence, $c_{t}^{*}$ is an affine function of $Y$. By substituting (A11) into (A10), we obtain:

$$
u_{t}=M^{(\beta-1) / \beta} \frac{(S \alpha+\beta Y)^{(\beta-1) / \beta}}{\beta-1} e^{(\beta-1)(r-\rho) t},
$$

where $S \equiv \frac{1-e^{-r T}}{r}$ is the annuity factor. Substituting (A12) into (1), finally, gives

$$
U=V(Y)=M^{(\beta-1) / \beta} \frac{(S \alpha+\beta Y)^{(\beta-1) / \beta}}{\beta-1} \int_{0}^{T} e^{(\beta-1)(r-\rho) t} e^{-\rho t} d t=M^{-1 / \beta} \frac{(S \alpha+\beta Y)^{(\beta-1) / \beta}}{\beta-1},(A
$$

showing that $V$ is indeed HARA in $Y$.

QED

\section{Proof of Proposition 2.}

One can obtain $\tilde{U}$ by multiplying the right hand side of (A13) by $M^{1 / \beta} S^{(1-\beta) / \beta}$, i.e. by applying an affine transformation. Alternatively, by continuity and the mean value theorem there must exist at least one point in time $\tau$, such that $c_{\tau}^{*}=c^{0}$, implying that Proposition 2 is just a special case of Proposition 1. QED

The Expo-power instantaneous utility function

The EP instantaneous utility function can be written as

$$
u_{t}=-e^{-\omega c_{t}^{\sigma}}
$$

so that

$$
u^{\prime}\left(c_{t}\right)=\omega \sigma c_{t}^{\sigma-1} e^{-\omega c_{t}^{\sigma}} .
$$


Hence, for $u_{t}$ to be increasing in $c_{t}$ we must impose the parameter restriction that $\sigma \omega>0$. It follows that the coefficients of absolute and relative risk aversion are given by $A_{t}=\frac{1-\sigma+\sigma \omega c_{t}^{\sigma}}{c_{t}}$, and $R_{t}=1-\sigma+\sigma \omega c_{t}^{\sigma}$, respectively. Hence the EP instantaneous utility function is characterized by CRRA in the limit case when $\omega=0$ approaches 0 , whereas $u$ is CARA when $\sigma=1$. Moreover, we observe DARA for $\sigma<1$, IARA for $\sigma>1$, DRRA for $\omega<0$, and IRRA for $\omega>0$. The EP instantaneous utility function is hence always concave for $\sigma<1$, while for $\sigma>1$ we need that $\omega c_{t}^{\sigma}>1-\frac{1}{\sigma}$. Moreover, and more importantly, unlike HARA the degree of risk aversion for the EP function is not globally monotonic in its parameter values. Indeed, $A_{t}$ (and of course $R_{t}$ as well) is increasing in $\sigma$ when $\omega c_{t}^{\sigma}\left(1+\sigma \ln c_{t}\right)>1$, and decreasing when the opposite holds. Likewise $A_{t}$ increases in $\omega$ for $\sigma>0$ and decreases for $\sigma>0$. These features make EP parameters less straightforward to interpret, and they may also make the numerical calculations more problematic.

By substituting (A15) into (3) we obtain

$$
u^{\prime}\left(c_{0}\right) e^{(\rho-r) t}=\omega \sigma c_{t}^{\sigma-1} e^{-\omega c_{t}^{\sigma}}
$$

for which there is no algebraic solution with respect to $c_{t}$, preventing us from obtaining a closed form solution of the optimal consumption path. Moreover, it can be shown by contradiction that optimal initial consumption cannot in general be written as an affine function of $Y$, implying that the functional form does in this case not carry over from $u$ to $V$; hence, $V$ will not have the expo-power functional form. ${ }^{21}$ The exception is when $\rho=r$ for

\footnotetext{
${ }^{21}$ Assuming that we can write $c_{0}^{*}=B^{1}+B^{2} Y$, we have by (A15) that $\frac{d V}{d Y}=\omega \sigma\left(B^{1}+B^{2} Y\right)^{\sigma-1} e^{-\omega\left(B^{1}+B^{2} Y\right)^{\sigma}}$. But if $V$ is expo-power so that we can write $V=-e^{-\omega Y^{\sigma}}$ we have that $\frac{d V}{d Y}=\omega \sigma Y^{\sigma-1} e^{-\omega Y^{\sigma}}$, which is inconsistent with the above expression unless $B^{1}=0$, for which initial consumption is proportional to $Y$. This solution follows from the special case where $\rho=r$.
} 
which it follows directly from (3) that the optimal consumption is constant over time, and hence proportional to $Y$ in each period. Consequently, when $\rho=r$ then $V$ is also expo-power, so that we can write

$$
V=-e^{-\omega Y^{\sigma}}
$$

Let us here for simplicity focus on this case. As before, we can express the expected utility of a lottery as a function of $Y$, or as a function of $c^{0}$, which here thus corresponds to a constant consumption level over time. When an individual is indifferent between two lotteries, I and II, we then have

$$
\sum_{i=1}^{n} p_{i}^{\mathrm{I}} e^{-\omega\left(c^{0}+x_{i}^{\mathrm{I}} / S\right)^{\sigma}}=\sum_{i=1}^{n} p_{i}^{\mathrm{II}} e^{-\omega\left(c^{0}+x_{i}^{\mathrm{II}} / S\right)^{\sigma}}
$$

From (A18) we can in principle solve for $\omega$ for a given value of $\sigma$, and vice versa, or solve for either $\omega$ or $\sigma$ for a specified relationship between them. However, the functional form implies that for extreme values of risk aversion, as we have here, the combination of an exponential and a power function makes the EP function very sensitive to small deviations in parameter values, which in turn makes it difficult for numerical calculations to converge. For example, in the neighborhood of the CRRA case we have that the EP function here implies that the number $e$ should be taken to the power of the product of two terms, where one goes to zero and the other is larger than 10,000 to the power of 19248 (for the low-stake lottery), i.e. larger than 10 to the power of $76992 .^{22}$

Still, assuming that $\omega$ and $\sigma$ have been identified for an individual we can again calculate how the same individual should choose between a safe and a risky option regarding all future income levels, based on these preferences. Given indifference between the options we can solve for $c^{L}$ in the same way as we did for the other functional forms, and as reported for the HARA case in Table 3, as follows:

\footnotetext{
${ }^{22}$ The get a little perception of the magnitude of this number, note that the number of atoms in the universe is estimated to be in the order of magnitude of 10 to the power of 80 .
} 


$$
c^{L}=\left(\frac{1}{\omega} \ln \frac{1-p}{e^{-\omega\left(c^{S}\right)^{\sigma}}-p e^{-\omega\left(c^{H}\right)^{\sigma}}}\right)^{1 / \sigma} .
$$

Focusing on the extreme case where the high income outcome implies an infinite consumption level, we have instead

$$
c^{L}=\left(\left(c^{S}\right)^{\sigma}+\frac{1}{\omega} \ln (1-p)\right)^{1 / \sigma} .
$$

Thus, we have derived a similar expression as in the HARA case for how much the individual would need in the unlucky outcome when the lucky outcome is infinite and obtained with probability $p$. 
Table 1. Calculated implicit parameters of absolute and relative risk aversion when people are indifferent between Options I and II, where the empirical results are taken from Holt and Laury (2002), for different cases.

\begin{tabular}{|c|c|c|c|c|c|c|}
\hline \multirow[t]{2}{*}{ Option I } & \multirow[t]{2}{*}{$\overline{\text { Option II }}$} & \multirow{2}{*}{$\begin{array}{l}\text { Fraction } \\
\text { choosing } \\
\text { I }\end{array}$} & \multirow{2}{*}{$\begin{array}{l}\text { Implicit parameter of } \\
\text { absolute risk aversion } \\
A \text { if indifference } \\
\text { between I and II }\end{array}$} & \multicolumn{3}{|c|}{$\begin{array}{l}\text { Implicit parameter of relative risk aversion } \\
R \text { if indifference between I and II }\end{array}$} \\
\hline & & & & EUI & $\begin{array}{l}\text { EUCT } \\
\text { base case }\end{array}$ & $\begin{array}{l}\text { EUCT } \\
r=500 \%\end{array}$ \\
\hline \multicolumn{7}{|c|}{ Low payoff lottery choices } \\
\hline $5 / 10$ of 2 USD, $5 / 10$ of 1.6 USD & $5 / 10$ of 3.85 USD, $5 / 10$ of 0.1 USD & $66 \%$ & 0.101 & 0.146 & 19248 & 202.8 \\
\hline $6 / 10$ of 2 USD, $4 / 10$ of 1.6 USD & $6 / 10$ of 3.85 USD, $4 / 10$ of 0.1 USD & $40 \%$ & 0.299 & 0.411 & 56735 & 597.7 \\
\hline $7 / 10$ of 2 USD, $3 / 10$ of 1.6 USD & $7 / 10$ of 3.85 USD, $3 / 10$ of 0.1 USD & $17 \%$ & 0.516 & 0.676 & 97980 & 1032.1 \\
\hline \multicolumn{7}{|c|}{ High payoff lottery choices } \\
\hline $5 / 10$ of 40 USD, $5 / 10$ of 32 USD & $5 / 10$ of 77 USD, $5 / 10$ of 2 USD & $81 \%$ & 0.005 & 0.146 & 962.8 & 23.15 \\
\hline $6 / 10$ of 40 USD, $4 / 10$ of 32 USD & $6 / 10$ of 77 USD, $4 / 10$ of 2 USD & $62 \%$ & 0.015 & 0.411 & 2838 & 45.24 \\
\hline $7 / 10$ of 40 USD, $3 / 10$ of 32 USD & $7 / 10$ of 77 USD, $3 / 10$ of 2 USD & $39 \%$ & 0.026 & 0.676 & 4900 & 69.75 \\
\hline
\end{tabular}

Note: $A$ is expressed in terms of the payoffs. All numerical calculations are performed in Mathematica.

Table 2. Calculated implicit annual consumption for the rest of life in the unlucky outcome based on the EUCT model, so that the degrees of risk aversion correspond to the ones obtained in Table 1.

\begin{tabular}{|c|c|c|c|c|c|c|}
\hline \multirow{4}{*}{$\begin{array}{l}\text { Safe option } \\
\text { USD/Year }\end{array}$} & \multicolumn{5}{|c|}{ Risky option } & \multirow{4}{*}{$\begin{array}{l}\text { Fraction } \\
\text { that would } \\
\text { choose the } \\
\text { safe option }\end{array}$} \\
\hline & \multirow{3}{*}{$\begin{array}{c}\text { Lucky outcome } \\
\text { (probability = 99\%) } \\
\text { USD/year }\end{array}$} & \multicolumn{4}{|c|}{ Unlucky outcome (probability =1\%), USD/year } & \\
\hline & & \multicolumn{2}{|c|}{ CARA } & \multicolumn{2}{|c|}{ CRRA } & \\
\hline & & $r=5 \%$ & $r=500 \%$ & $r=5 \%$ & $r=500 \%$ & \\
\hline & \multicolumn{6}{|c|}{ Based on low payoff lottery choices } \\
\hline 36,000 & Infinite & 35999.0 & 35901 & 35991 & 35188 & $66 \%$ \\
\hline 36,000 & Infinite & 35999.6 & 35967 & 35997 & 35723 & $40 \%$ \\
\hline 36,000 & Infinite & 35999.8 & 35981 & 35998 & 35840 & $17 \%$ \\
\hline & \multicolumn{6}{|c|}{ Based on high payoff lottery choices } \\
\hline 36,000 & Infinite & 35979 & 34000 & 35828 & 29242 & $81 \%$ \\
\hline 36,000 & Infinite & 35993 & 35333 & 35942 & 32441 & $62 \%$ \\
\hline 36,000 & Infinite & 35996 & 35615 & 35966 & 33668 & $39 \%$ \\
\hline
\end{tabular}


Table 3. Calculated implicit annual consumption for the rest of life in the unlucky outcome based on the EUCT model with HARA preferences, based on the experimental choices reported by Holt and Laury (2002), as reported by the first and the fifth pairwise choices in Table 1 in this paper. The parameter $1 / \beta$ is reported in brackets, so that $A$ and $R$ can easily be calculated for different consumption levels.

\begin{tabular}{|c|c|c|c|c|}
\hline$\alpha / \beta$ & $\begin{array}{l}\text { Outcome in safe } \\
\text { option } \\
\text { USD/Year }\end{array}$ & $\begin{array}{c}\text { Lucky outcome } \\
\text { (probability }=99 \%) \\
\text { USD/year }\end{array}$ & $\begin{array}{l}\text { Unlucky outcome } C^{L} \text { (probability }=1 \% \text { ) based } \\
\text { on low payoff lottery choices; } 66 \% \text { would } \\
\text { choose the safe option }\end{array}$ & $\begin{array}{l}\text { Unlucky outcome } C^{L} \text { (probability }=1 \% \text { ) based } \\
\text { on high payoff lottery choices; } 62 \% \text { would } \\
\text { choose the safe option }\end{array}$ \\
\hline & & & $r=500 \%$ & $r=500 \%$ \\
\hline \multirow[b]{2}{*}{$-\infty$} & \multicolumn{4}{|c|}{$\underline{u}$ characterized by CARA and IRRA } \\
\hline & 36,000 & Infinite & $35901[-\infty]$ & $35993[-\infty]$ \\
\hline & \multicolumn{4}{|c|}{$\underline{u}$ characterized by IARA and IRRA } \\
\hline-500000 & 36,000 & 100,000 & $35785[-192474]$ & $35985[-139010]$ \\
\hline-200000 & 36,000 & 100,000 & $35998[-365700]$ & $35986[-53902]$ \\
\hline-110000 & 36,000 & 100,000 & $35998[-192474]$ & $35988[-28369]$ \\
\hline \multicolumn{5}{|c|}{$u$ is DARA and DRRA and chosen such that the choices based on the EUCT model with $c^{0}=10,000$ coincides with the choices based on the EUI model } \\
\hline$-10,000$ & 36,000 & 36,200 & $17619[0.146]$ & $19496[0.411]$ \\
\hline \multicolumn{5}{|c|}{$u$ is DARA and DRRA and chosen consistent with the median subject in both the small-stake and the large-stake experiments of Holt and Laury, $r=5 \%$} \\
\hline-9999.84 & 36,000 & 36,200 & $19878[0.479] \ldots \ldots$ & $19878[0.479]$ \\
\hline \multicolumn{5}{|c|}{$u$ is DARA and DRRA and chosen consistent with the median subject in both the small-stake and the large-stake experiments of Holt and Laury, $r=500 \%$} \\
\hline-9964.41 & 36,000 & 36,200 & $21825[0.906]$ & $21,825[0.906]$ \\
\hline \multicolumn{5}{|c|}{$u$ characterized by DARA and DRRA } \\
\hline-9900 & 36,000 & Infinite & $35380[193]$ & 9900 [1.22] \\
\hline-9000 & 36,000 & Infinite & 35935 [1925] & 35565 [284] \\
\hline-7000 & 36,000 & Infinite & $35977[5774]$ & $27438[14.2]$ \\
\hline-4000 & 36,000 & Infinite & $35987[11549]$ & $35914[1703]$ \\
\hline \multicolumn{5}{|c|}{ u characterized by DARA and CRRA } \\
\hline 0 & 36,000 & Infinite & $35991[19248]$ & $35942[2838]$ \\
\hline \multicolumn{5}{|c|}{ u characterized by DARA and IRRA } \\
\hline 5000 & 36,000 & Infinite & $35993[28871]$ & $35956[4256]$ \\
\hline 20000 & 36,000 & Infinite & $35996[57742]$ & $35970[8511]$ \\
\hline 50000 & 36,000 & Infinite & $35997[115485]$ & 35977 [17022] \\
\hline 200000 & 36,000 & Infinite & $35997[404196]$ & $35982[59577]$ \\
\hline \multicolumn{5}{|c|}{$u$ characterized by CARA and IRRA } \\
\hline$\infty$ & 36,000 & Infinite & $35901[\infty]$ & $35333[\infty]$ \\
\hline
\end{tabular}

